\title{
AN EFFICIENT FPGA BASED SPACE VECTOR PULSE WIDTH MODULATION IMPLEMENTATION FOR SERVO CONTROL APPLICATION
}

\author{
M. Kalpana ${ }^{1}$, G. Arumugam ${ }^{2}$ \\ ${ }^{I}$ P.G. Student, Department of ECE, Seshachala Institute of Technology, Puttur, AP, India \\ ${ }^{2}$ Assistant Professor, Department of ECE, Seshachala Institute of Technology, Puttur, AP, India
}

\begin{abstract}
This paper focuses on the design of a low power and high performance FPGA based Space Vector Pulse Width Modulation (SVPWM) controller for three phase implementation for Servo control Application. A new method is proposed to realize easy, accurate and high performance SVPWM technique based on FPGA with low resource consumption and reduced execution time than conventional methods. The FPGA based SVPWM generation involves a digital controller implementation to execute the algorithm and $D Q$ reference generator gives reference vector signal to the controller. The controller in turn controls the PLL generator to generate pulse width modulated clock for three phases. The PLL generator is sourced by clock generator. Experimental results are presented for SVPWM architecture synthesized on standard low-cost FPGA chips, showing very good linearity and resolutions up to Ins.
\end{abstract}

Keywords: FPGA, PLL Generator, Space Vector Pulse Width Modulation (SVPWM)

\section{INTRODUCTION}

An analog signal has a continuously varying value, with infinite resolution in both time and magnitude. By controlling analog circuits digitally, system costs and power consumption can be drastically reduced. What is more, many microcontrollers and DSPs already include on-chip PWM controllers, making implementation easy. PWM is a way of digitally encoding analog signal levels. Digital control has obtained great research attention due to their advantages, such as low sensitivity, advanced control algorithms, reduced component count, ease of design and prototyping, etc. The advantage of these DPWMs is that they are very simple and obtain high linearity. However, their resolution cannot be very high, as the minimum time step is equal to the clock period of the counter. Furthermore, their power consumption is proportional to the clock frequency; so, trying to obtain a relatively high resolution results in high power consumption. In order to increase DPWMs' resolution, delay lines can be used.

This paper describes FPGA base SVPWM architecture, this involves digital controller implementation which will execute the algorithm. The SVPWM technique is very popular in three phase systems due to its performance benefits. There are many ways of Pulse width modulation schemes to obtain variable voltage and frequency supply. The widely used Pulse Width Modulation is Space Vector Pulse Width Modulation due to its due to its wide range of applications. SVPWM has low harmonic distortion, less hardware and low power consumption.
In this paper low cost Field Programmable Gate Array (FPGA) based design of SVPWM generation involves a digital controller implementation to execute the algorithm and DQ reference generator gives reference vector signal to the controller. The controller in turn controls the PLL generator to generate pulse width modulated clock for three phases. The PLL generator is sourced by clock generator.

\section{EXISTING SYSTEM}

The architecture of PLL-based DPWM is shown in Fig. 1. The PLL-based DPWM will generate fixed delays and it will avoid post fitting delay adjustments. The Digital controller in turns control the generator to generate pulse width modulated clock for phases. The Digital Pulse Width Modulation can manage the clock signals given by the PLLs. We can change the phases of clock signals and we can manage the frequencies and we can control them in a specified manner. The PLL can multiply the generated clock frequency from $25 \mathrm{MHz}$ to $250 \mathrm{MHz}$. We can generate additional outputs P90, $\mathrm{P} 180$ and $\mathrm{P} 270$ with main output P0. The signals will generate high resolution of the DPWM, adding the other input bits provides adjustment of the duty cycle will reach 1 ins resolution.

The variations in delay due to different paths inside the multiplex or may lead to non monotonic non linear behavior. To eliminate these effects calibration process will be used. The post-fitting simulation has to run for estimating the delay differences and the phase shifts at the PLL outputs are adjusted accordingly. The digital phase-locked loop (DPLL) has better controlling over the analog PLL, PID, fuzzy logic controller etc. and so it provides accurate speed control on 
loading. DPLL is used in various fields such as communication fields, instrumentation and control fields etc. to escape the drawbacks such as the various types of noise like white noise, spur noise, damping factor, high frequency noise etc. get introducing with linear PLL, it has also poor stability of locking range of frequency so dumping occurs and less efficient for the higher frequencies. Digital PLL controls the speed of DC motors and provides wide locking range. The Digital PLL provides better synchronization for digital signals with help of phase frequency detector and loop filter.
Delay differences due to different paths inside the multiplexer may lead to non-monotonic and non-linear behavior. These undesired effects are eliminated by a calibrating procedure: a post-fitting simulation is run to estimate the delay differences and the phase shifts at the PLL outputs are adjusted accordingly. Special care must be taken to lock on-chip placement of the DPWM module resources after this calibration is completed. Otherwise the insertion of additional circuit elements can completely modify the placement of the circuit inside the chip and therefore also the timing and delays, leaving the previous calibration useless.

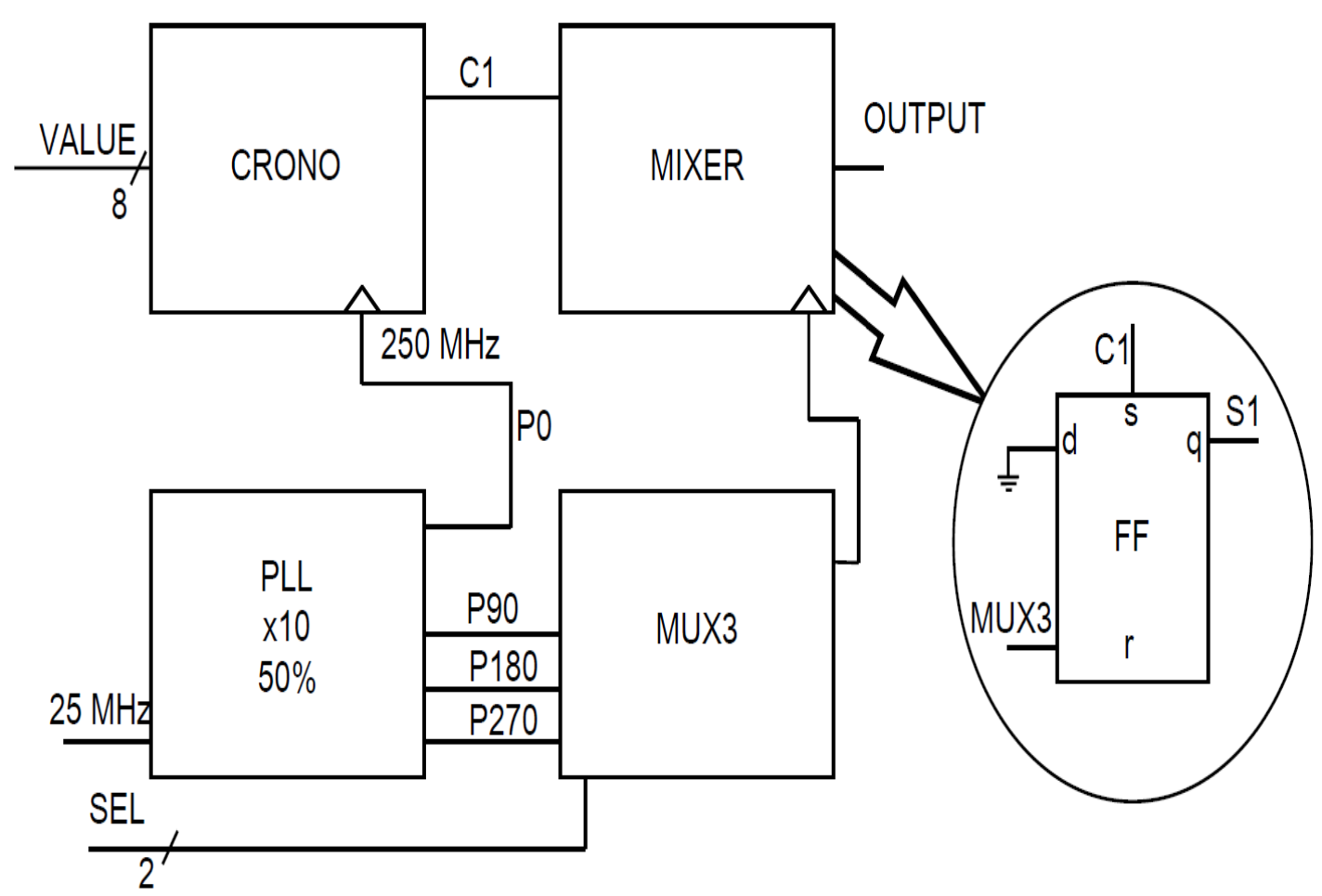

Fig. 1 Simplified diagram of the PLL-based DPWM architecture

\section{PROPOSED SYSTEM}

Pulse-width modulation (PWM), or pulse-duration modulation (PDM), is a technique used to encode a message into a pulsing signal. Space vector modulation (SVM) is an algorithm for the control of pulse width modulation (PWM).It is used for the creation of alternating current (AC) waveforms, most commonly to drive 3 phase AC powered motors at varying speeds from DC using multiple class-D amplifiers. There are various variations of SVM that result in different quality and computational requirements. The design of Efficient FPGA based Digital Space Vector Pulse Width Modulation controller for Servo control Application. The method involves SVPWM generation.

The FPGA based SVPWM generation involves a digital controller implementation to execute the algorithm and DQ reference generator gives reference vector signal to the controller. The controller in turn controls the PLL generator to generate pulse width modulated clock for three phases. The PLL generator is sourced by clock generator
The SVPWM technique offers significant performance benefits and has proved to be very popular in three-phase systems such as Servo Controllers, Power Converters and Robotics. The system is having many advantages such as SVPWM based control of power drives gives precision control, Low Harmonic Distortion compared to other PWM techniques and reduction of hardware etc. 


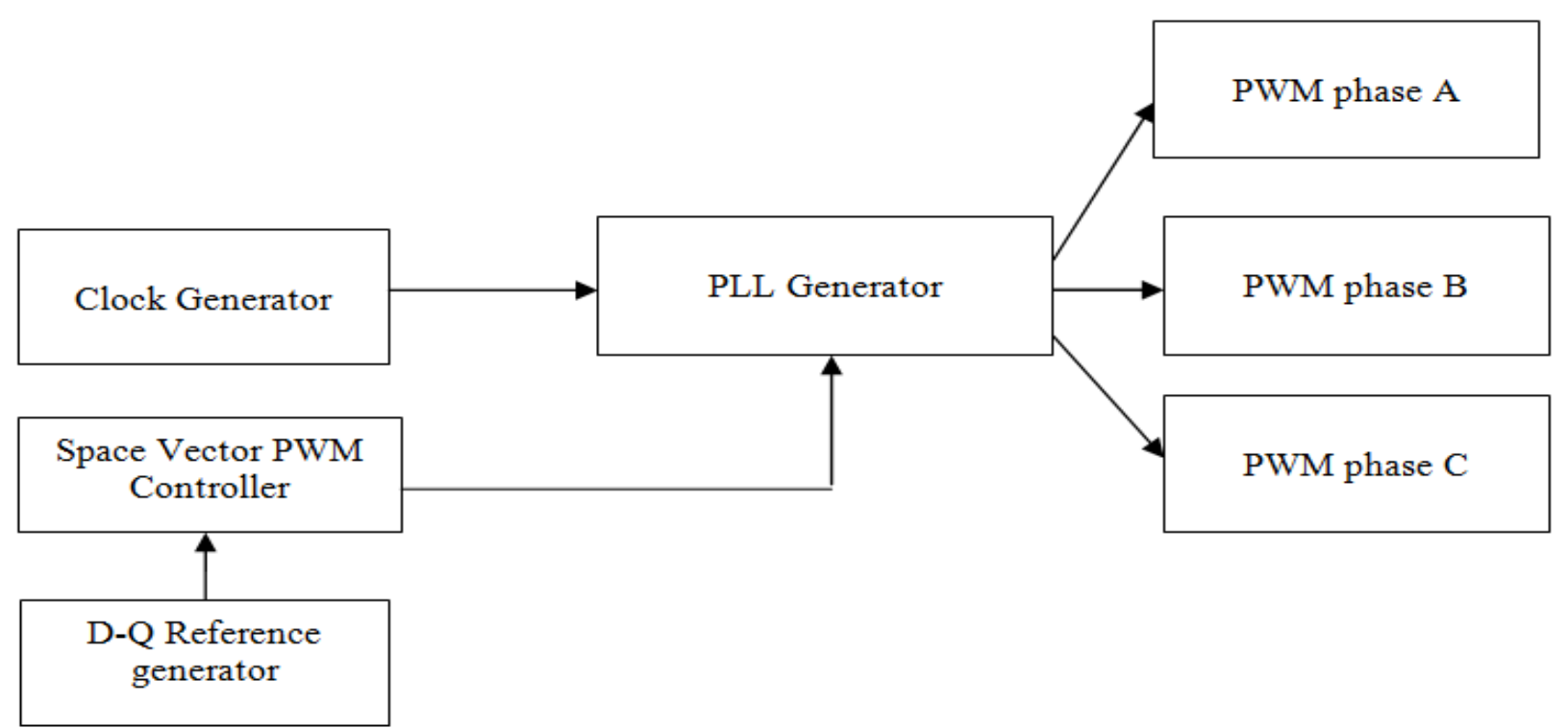

Fig 2: Block diagram for Efficient FPGA based Space vector pulse width modulation implementation for Servo control Application

The FPGA based SVPWM generation involves a digital controller implementation to execute the algorithm and DQ reference generator gives reference vector signal to the controller. The controller in turn controls the PLL generator to generate pulse width modulated clock for three phases. The PLL generator is sourced by clock generator. The based SVPWM generation having following advantages

- SVPWM based control of power drives gives precision control.

\section{SIMULATION RESULTS}

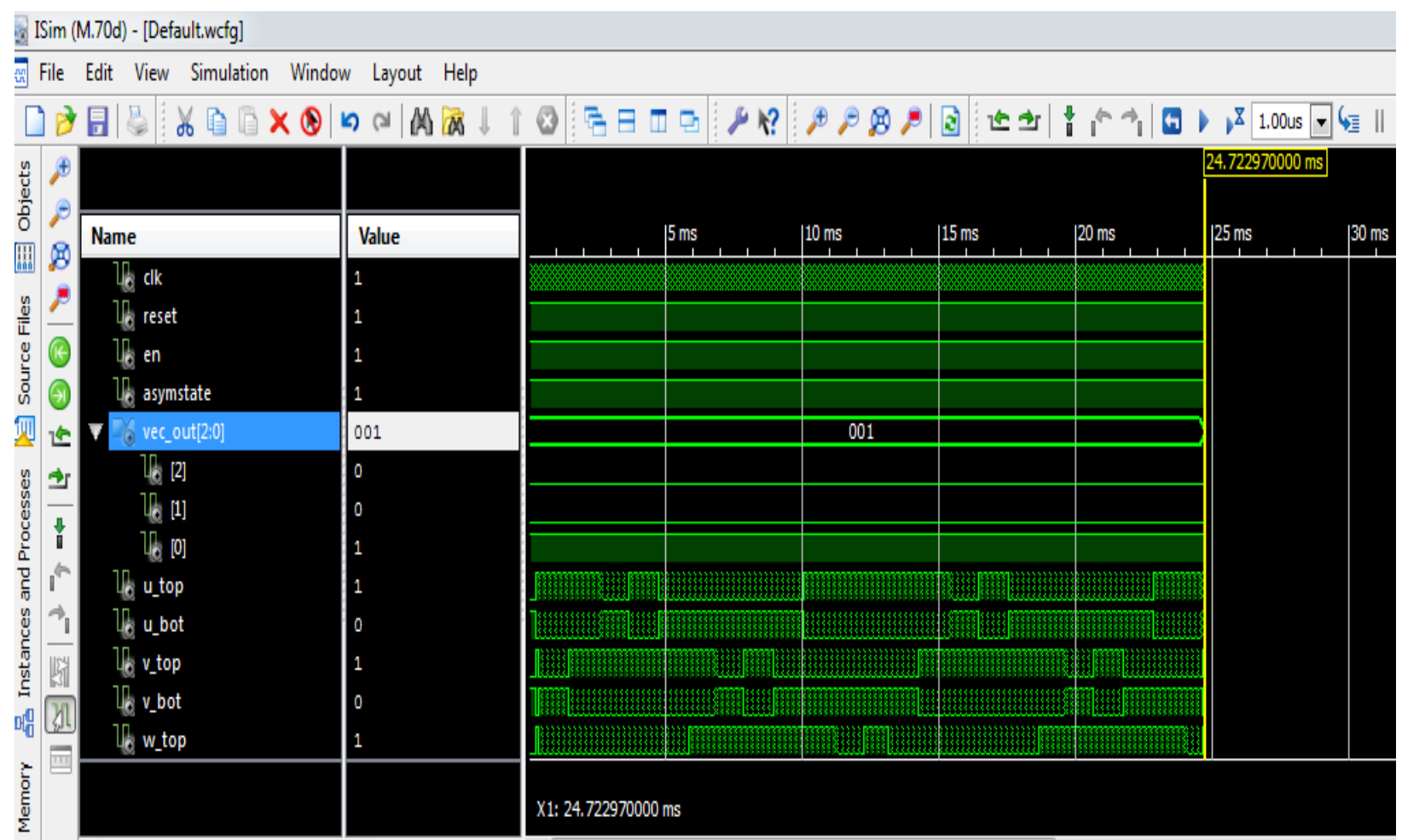

(a)
- Low Harmonic Distortion compared to other PWM techniques.

- $\quad$ FPGA based SVPWM generation implies reduction of Hardware and can be implemented to generate dynamic control algorithm with less power consumption. 


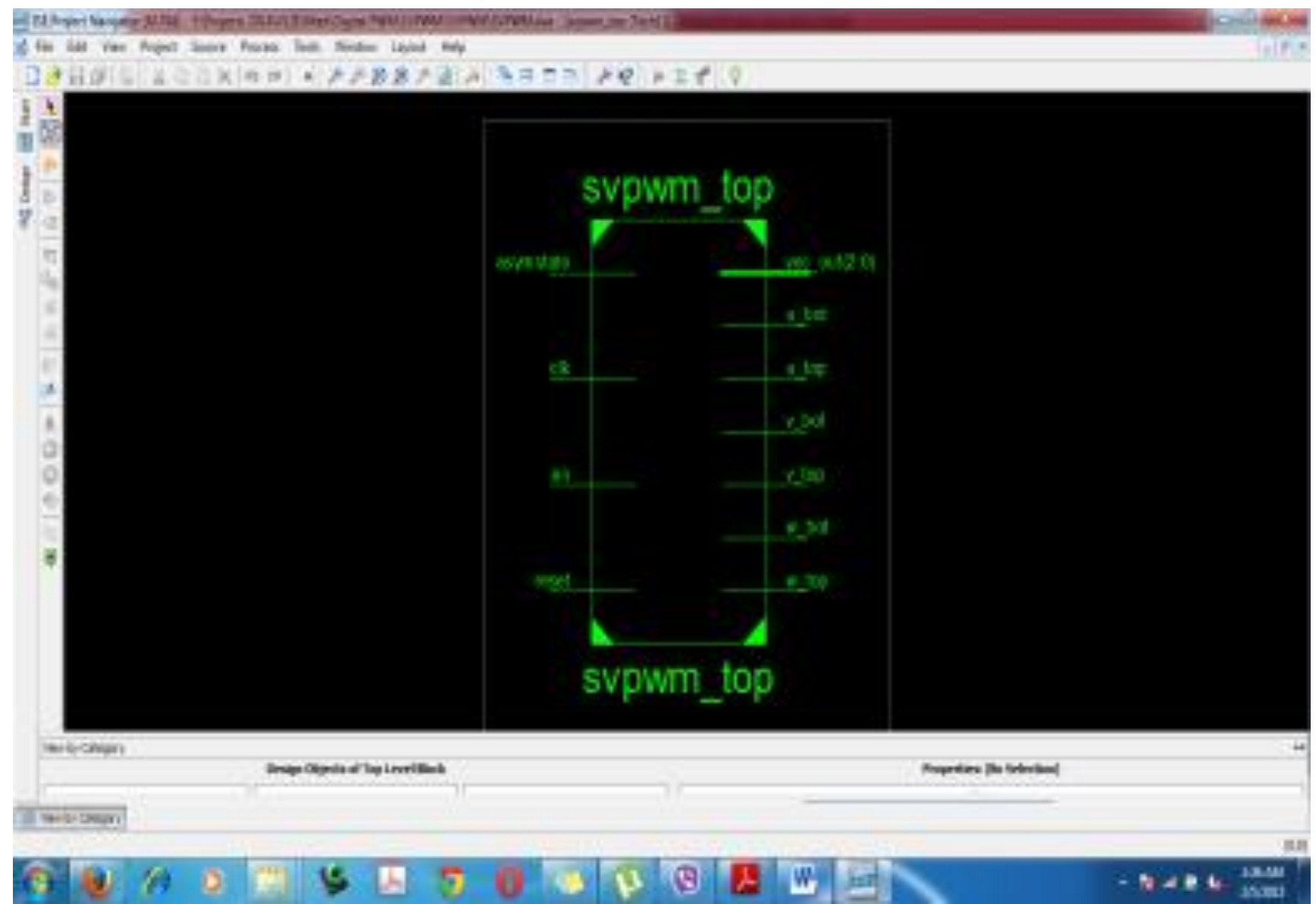

(b)

Fig 2 Simulation results (a) Simulation output (b) Space Vector Pulse Width Modulation

Above Figures shows the results of FPGA based Space Vector Pulse Width Modulation Simulation results as well as top module of Space Vector Pulse Width Modulation.

\section{Timing Analysis Report}

Data Sheet report:

All values displayed in nanoseconds (ns)

Setup/Hold to clock clk

|Max Setup to|Max Hold to | Clock |

Source $\mid$ clk (edge) $\mid$ clk (edge) |Internal Clock(s) | Phase | asymstate | $0.283(\mathrm{R})|\quad 0.405(\mathrm{R})| \mathrm{clk}$ BUFGP 0.000

\begin{tabular}{|c|c|c|}
\hline en & $0.280(\mathrm{R})$ & 0.545(R)|clk_BUFGP \\
\hline reset & $1.579(\mathrm{R})$ & 0.545(R)|clk_BUFGP \\
\hline
\end{tabular}

Clock clk to Pad

-------------+-------------+--------------------+---------+

| clk (edge) | |Clock |

Destination | to PAD |Internal Clock(s)| Phase |

\begin{tabular}{l|l|l} 
u_bot & $6.216(\mathrm{R}) \mid \mathrm{clk}$ _BUFGP & 0.000 \\
u_top & $6.216(\mathrm{R}) \mid \mathrm{clk}$ _BUFGP & 0.000
\end{tabular}

\begin{tabular}{|c|c|c|}
\hline v_bot & 6.227(R)|clk_BUFGP & 0.000 \\
\hline v_top & $6.227(\mathrm{R}) \mid \mathrm{clk}$ _BUFGP & 0.000 \\
\hline vec_out $\langle 0\rangle$ & 6.216(R)|clk_BUFGP & 0.000 \\
\hline vec_out $\langle 1\rangle$ & $6.227(\mathrm{R}) \mid \mathrm{clk}$ _BUFGP & 0.000 \\
\hline vec_out $\langle 2\rangle$ & 6.216(R)|clk_BUFGP & 0.000 \\
\hline w_bot & 6.216(R)|clk_BUFGP & 0.000 \\
\hline w_top & 6.216(R)|clk_BUFGP & 0.000 \\
\hline
\end{tabular}

Clock to Setup on destination clock clk

| Src:Rise| Src:Fall| Src:Rise| Src:Fall|

Source Clock |Dest:Rise|Dest:Rise|Dest:Fall|Dest:Fall|

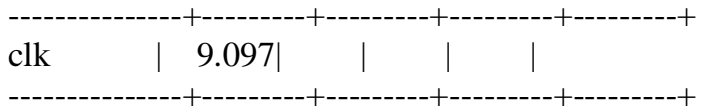

Analysis completed Thu Feb 05 01:42:49 2015

Trace Settings:

Trace Settings

Peak Memory Usage: 119 MB 


\section{CONCLUSION}

FPGA based Space vector PWM implemented, which involves digital controller implementation. The digital controller executed the algorithm for reference vector signal to the controller. The PLL generator generated pulse width modulation clock for three phases. The SVPWM based method achieves a resolution of $1 \mathrm{~ns}$ in the low-cost FPGA It is concluded that solution have good linearity and monotonicity properties.

\section{REFERENCES}

[1] Prodic, D. Maksimovic, and R. W. Erickson, "Design and Implementation of a DigitalPWM Controller for a High-Frequency Switching DC-DC Power Converter," The $27^{\text {th }}$ Annual Conference of the IEEE Industrial Electronics Society, IECON 2001, Vol.

[2] R.F. Foley, R.C. Kavanagh, W.P. Marnane and M.G. Egan, "A versatile digital pulsewidth modulation architecture with area-efficient FPGA implementation." Power Electronics Specialists Conference, PESC 2005.

[3] V. Yousefzadeh, T. Takayama, D. Maksimovic. "Hybrid DPWM with digital delay-locked loop." IEEE Computer in Power Electronics Workshop, COMPEL 2006.

[4] S.C. Huerta, A. de Castro, O. García, J.A. Cobos. "FPGA based digital pulse width modulator with time resolution under 2 ns." IEEE Tran. on Power Electronics, Vol. 23, No. 6, November 2008.

[5] Óscar López, Jacobo Álvarez, Jesús Doval-Gandoy, and Francisco D. Freijedo, "Multilevel Multiphase Space Vector PWM Algorithm", IEEE TRANSACTIONS ON INDUSTRIAL ELECTRONICS, VOL. 55, NO. 5, pp. 1933-1942, MAY 2008. 